\title{
The Twilight of Automation
}

\author{
Mario Khreiche
}

\section{Introduction: (Semi) Automation and the Future of Work}

The future of work has come under renewed scrutiny amidst growing concerns about automation threatening widespread joblessness and precarity. While some researchers rush to declare The Second Machine Age (Brynjolfsson and McAfee 2014), The Rise of the Robots (Ford 2016), or The Fourth Industrial Revolution (Schwab 2016), others proceed with business as usual, suggesting that specialized job training and prudent reform will sufficiently equip workers for future employment (Atkinson and Wu 2017). Among the points of contention are the scope and rate whereby human labor will be replaced by machines. Inflated predictions in this regard not only entice certified technologists and neoclassical economists (Sundararajan 2016), but also increasingly sway leftist commentators who echo the experts' cases for ramping up the proliferation of network technologies and accelerating the rate of automation in anticipation of a postcapitalist society (see Srnicek and Williams 2016, Mason 2017, Frase 2016, Rifkin 2015). In this essay, however, I caution that under the current cultural dictate of relentless self-optimization, ubiquitous economic imperatives to liquidate personal assets, and nearly unbridled corporate ownership of key infrastructures in communication, mobility, and, importantly, labor itself, an unchecked project of automation is both ill-conceived and ill-fated. Instead, the task at hand is to provide a more detailed account on the nexus of work, automation, and futurizing, to formulate a challenge to the dominance of techno-utopian narratives and intervene in programs that too readily endorse the premises and promises of fully automated futures.

As it stands, automation unfolds unevenly across socioeconomic domains, thus exacerbating precarity for workers in only partially automated systems while stealthily increasing wealth and power for service providers. Though automation processes take place in virtually all industries, the phenomenon of the so-called gig economy indicates how automated services rest primarily on workers' willingness to temporarily render their time, labor, and property available for other network participants. In these highly deregulated markets, some emerge as employers, makers, and coders while others are rendered logistical assets that complete the codes of the new modes of mobility, housing, and digital work. Gig-economy firms obscure information asymmetries and vertical power relations in their applications, offering in return narratives whereby robotized workers can consider themselves as entrepreneurial as the platform startups, whose innovative spirits they emulate. Thus, the confidence placed in current forms of automation, particularly regarding their potential to increase leisure time and economic freedom, is severely misplaced. Instead, today's digitally-mediated services conceal human labor and spin tales of user-entrepreneurialism to appease investors who, in turn, sponsor calculated business plans that trade instant profits for long-term market share. Guised as automation, the gig economy embodies a trajectory in which growing corporate power coincides with considerable reductions of employment rights and benefits for workers. The veneer of automation serves to advance monopolistic conditions by programming gigification, gamification, and taskification into the circuits of social, cultural, and economic exchange. Therefore, application interfaces contain shifting meanings of value accumulation that act as subtle yet powerful forces. What is ultimately considered acceptable, feasible, even meaningful economic activity, meanwhile, remains irreducible to technological forms.

Contrary to the narrative of automation as a mere job-eliminating force, this essay gestures toward a twilight of automation, where work is less replaced than displaced. To support this view, I foreground the concept of continuity as a programming feature in gig-economy interfaces and as a mode of engagement that perpetually shifts 
the social meanings of economic activity. Such an intervention at the intersection of technology, culture, and work is crucial given the myriad of recent publications in so-called postcapitalist discourses that fail to attend to the implications of reconfigured work processes and corresponding social relations. Postcapitalist commentators jettison a detailed discussion of digital economies, where providers manipulate workers' senses of time, space, and self to optimize service distribution. A critical perspective of automation in the gig economy elucidates the demands of constant availability, connectivity, and communicability that push workers to conform their identity and immediate surrounding to the injunctions of late capitalism.

Insights from three popular gig economies - the ride-hailing service Uber, the home-sharing application Airbnb, and the micro labor brokerage Amazon Mechanical Turk (AMT) — serve to critique several postcapitalist projects: Nick Srnicek and Alex Williams' Inventing the Future: Postcapitalism and a World Without Work (2016), Paul Mason's Postcapitalism: A Guide to Our Future (2017), and Peter Frase's Four Futures: Life After Capitalism (2016). Extending the econofiction genre, I emphasize that projects of automation are contingent on sociotechnical machines and the production of entrepreneurial subjectivities.

\section{The Interfaces of Uber, Airbnb, and Amazon Mechanical Turk}

According to estimates, gig-economy workers make up anywhere between $0.5 \%$ and $16 \%$ of the United States workforce (Katz and Krueger 2015). Compared to the current spotlight, the number of people earning a steady income through Uber, Lyft, Airbnb, TaskRabbit, and other ventures remains relatively low (Vinik 2018). Contrary to self-referential allusions to its disruptive potential, the gig economy instead surfs a decades-old wave of deregulation policies and flexibilization of working conditions (Boltanski and Chiapello 2007; Sennett 2006). Workplaces are restructured around screens that mediate, monitor, and surveil. As media theorist Jonathan Crary notes, "The individual [...] is constantly engaged, interfacing, interacting, communicating, responding, or processing within some telematic milieu" (Crary 2014: 15). Even the gig economy's most ardent critics often fail to recognize the historical context for the deluge of interfaces promising "to do more with less," reproducing the hubris of technological exceptionalism (see Fowler 2018). Despite caveats of scale and novelty, the gig economy must be taken seriously as a manifestation of a trend toward informalized and unremitting work relations. Indeed, the fuzzy employment statistics encapsulate the rise of nonconventional work arrangements, challenging widely-held assumptions about what counts as meaningful economic activity. In this view, efforts to measure the effects of automation by appraising the rate whereby tasks in a profession become automatable, rather than accounting for professions per se, emphasize the need to consult qualitative employment accounts (Arntz, Gregory, and Zierahn 2016). Perhaps inadvertently, the focus on tasks as the primary metric in assessing the impacts of automation indicates a shift toward the economization of all activities, underscoring the ubiquitous demand to engage with multiple market interfaces.

The economies of Uber, Airbnb, and AMT exhibit this trend in their streamlined communication with users on the supply side, respectively, drivers, home sharers, and micro laborers. Encouragements to work extra hours, rent additional space, and complete more tasks, as the case may be, materialize in messages, notifications, and updates. Notably, economic activity in the gig economy increasingly registers as a hybrid of work and play. As media critic Alexander Galloway observes, these domains have become virtually inseparable: "labor itself is now play, just as play becomes more and more laborious" (Galloway 2012: 29). Indeed, gig-economy interfaces commonly issue gamified incentives, customized around users' relation to the marketplace. For instance, novice or casual Lyft or Uber drivers are likely to receive more valuable bonuses than frequent drivers who are merely reminded of their set financial goals (Mason 2018). Should drivers wish to clock out before the company limit, currently set at twelve hours (Farivar 2018), Uber might relay prompts including a graphic of a gauge with a needle just shy of a maximum value and a text that reads, “You're \$10 away from making \$330 in net earnings. Are you sure you want to go offline?” (Scheiber 2017). Beyond titillating drivers' entrepreneurial sensibilities, either by positive trigger or fear of missing out on potential earnings, the firm rigorously collects data to optimize its service. If a driver wishes to be at a certain place at a certain time, Uber might suggest fares that steadily guide the driver closer to the final destination (Ibid.).

Aside from more or less subtle incentives, the incorporation of humans into the itineraries and virtual projections of gigified mobility influences driver subjectivity in psychological and behavioral terms. The indisputable efficacy of Uber's reward and rating systems; for instance, noticeably affect drivers' demeanors. As private cars turn into commercial assets, physical space between driver and passenger is commodified according to Uber's community 
guidelines that serve to enforce smooth market relations. In addition to defaulting to silent chauffeur mode, many drivers invest in headrest display cards to solicit optimal ratings, badges, and tips or offer amenities, such as bottled water, chewing gum, or phone charge. The cultural acceptance of rideshare platforms and the behavioral codes therein are less imposed by service providers than cogenerated and normalized over time through social habits and interactions in online and offline communities.

Typically, rideshare interfaces communicate a kind of endless temporality, a technologically mediated sense that the future is already contained in the present moment. The design is often so sleek that passengers virtually arrive as soon as they open the application. Paul Virilio considers this phenomenon as an aesthetics of disappearance in which "there will be no longer anything but arrival, the point of arrival, the departure will itself have disappeared in the instantaneity of the projection" (Virilio 2008: 11). The conditions that collapse present and future exceed the interface construed as a material device. Thus, the task is, in Galloway's words, "to identify the interface itself as historical" (Galloway 2012: 30). To anticipate a central criticism of postcapitalist futurizing, the notion that access, speed, and continuity necessarily translate to more freedom is refuted in a Deleuzian sense whereby one "is never finished with anything" (Deleuze 1992: 4-5). The forms of "always-arriving-but-never-ending" accumulation materialize in the gig economy and inform a culture of uninterrupted engagement with interfaces as a coherent representation of economic activity.

While Uber is in the business of frictionless (auto)mobility, Airbnb deals in on-demand lodging. To connect hosts and guests more seamlessly to the platform, the firm continuously improves its design: "Hosts [...] need to track earnings, get rooms cleaned on time, and provide a de facto concierge service" (Rhodes 2015). Application updates organize information more efficiently, increase access, and decrease communication latencies (Perez 2016). These changes aim to produce efficiently performing gig-economy subjectivities, competitive entrepreneurs of the self unphased by the underlying codes that differentiate success in the same marketplace. Indeed, under the veil of open-minded, worldwide communities and all-around desirable disruption of international travel, Airbnb runs a deeply irregular business operation.

Since 2015, Airbnb encourages hosts to aspire to its so-called superhost status. Requirements to receive a superhost badge stipulate hosting at least 10 trips per year, maintaining a minimum $90 \%$ response rate, and achieving an $80 \%$ share of five-star ratings. Though technically anyone might qualify for a superhost badge, only about $7 \%$ of users make the cut (Chen 2017). Benefits include higher search visibility, invites to exclusive Airbnb events, and increased market credibility. Alternative strategies to boost market efficiency involve bots responding to guest inquiries overnight to improve communication. The commodification of time in these systems constitutes the new norm. Users may opt for a more casual approach to Airbnb, but the imperatives of constant availability saturate the entire marketplace. The injunction for on-demand responsiveness characterizes Crary's $\underline{24 / 7}$, a tale of an insomniac subjectivity "shaped around individual goals of competitiveness, advancement, acquisitiveness, personal security, and comfort at the expense of others" (Crary 2014: 41).

Luxury management services, such as Happy Host and MetroButler, optimize the Airbnb experience for those who can afford it. These agencies deploy software tools to assess the value of real estate, handle bookings, hire cleaners, and take on tedious tasks for clients in high-margin neighborhoods. Management services illustrate the gig economy's complicity in integrating routine low-income professions into the logic of crowdsourced labor distribution, a certified approach to eliminating overhead costs and attracting outside capital. The trend toward evermore moving parts to be reorganized in a profit-oriented application results in the continuous displacement of workers, whose jobs always assume new meanings, as columnist Nathan Heller explains:

[A MetroButler worker] had put fresh company linens on the queen-size bed, and had left hotel-size shampoo and conditioner bottles, with the MetroButler logo, on the nightstand. He discovered that the bulb in the desk lamp had burned out, so he made a note to buy a replacement. [...] Every task was annotated on a photo of the space in an app that let MetroButler watch his progress in real time (Heller 2016).

Displacement and the crowding-out effect invite relatively privileged individuals to accept such gigs while workers who rely on more stable work relations and benefits are marginalized. Thus, Airbnb and its startup progenies foster competition on various levels: hosts must adjust pricing and increase service efforts through reduced response times and amenities. These demands spawn secondary economies that, in turn, informalize peripheral markets.

Gigification and continuous workflows also characterize Amazon's Mechanical Turk service, a data clearinghouse connecting clients (Requesters) to workers (Taskers or Turkers). AMT, according to its website, "operates a marketplace for work that requires human intelligence [and] enables companies to programmatically access this 
marketplace and a diverse, on-demand workforce." Humans still outperform computers in tasks, such as identifying and classifying objects in images and videos, finding data duplicates, and transcription. These services attract expense management businesses, such as Expensify, which enlists AMTs contingent workforce to transcribe customer receipts while pretending to use "smartscan technology" (Solon 2018). In addition, AMT serves as a viable tool for content moderation on video platforms, streaming services, and social media. Lastly, AMT is popular in the social sciences, because it enables researchers to cut time and costs of conducting academic surveys. In all these areas, AMT's wager is to render "human intelligence tasks" (HITs) accessible, scalable, and cost-effective. AMT charges a $20 \%$ fee for each transaction and an extra 20\% fee for HITs including at least 10 assignments. The marketplace lists about 500,000 tasks per day, though these figures vary significantly (Katz 2017).

AMT's design fittingly represents the role most Amazon workers play in the vast systems of cloud computing, networked automation, and uninterrupted consumer convenience. The brokerage advertises "access to thousands of high quality, global, on-demand Workers" who are kept at the backend of the interface, prefiguring their presence in a twilight economy of automation and residual human employability. If the partitioning of labor processes into userclients and user-laborers is not a particularly new phenomenon, the effects of removal and alienation in AMT are intensified, since the concealment of labor is as crucial as the labor itself “The interface," as David Berry observes, "reifies the social labour undertaken behind the surface, such that the machinery may be literally millions of humans 'computing' the needs to the software, all without the user being aware of it" (Berry 2015). Thus, "With workers hidden in the technology, programmers can treat workers like bits of code and continue to think of themselves as builders, not managers" (Irani: 2016: 36). Both descriptions echo Galloway's account of the interface as corporeal and incorporeal, material and ideological. The different designs and user interfaces for Turkers and Requesters, respectively, embody a historical configuration whereby digital work is part and parcel of an economic a system that sets out to eradicate its own reliance on labor.

Operating behind the scenes of big technology companies, information brokers, and academic research, AMT's legal meandering and quasi-exploitation hardly register in the same way that Uber and Airbnb continue to ruffle the feathers of regulators. While drivers and home sharers in the gig economy increasingly receive public attention, the sparsely paid gigs in AMT remain largely unnoticed. Likening AMT to a digital sweatshop, one commentator notes:

Many of the labor activists and scholars I spoke to for this story had never heard of Amazon Mechanical Turk, nor had several of the tech employees I reached out to-even ones who work at companies that employ microtaskers by the hundreds of thousands. Like Google Books employees, microtaskers are, for the most part, invisible. And that makes them easy to ignore (Cushing 2013).

Despite qualitative differences between platforms, Jeff Bezos' coinage of "artificial artificial intelligence" constitutes the engine in recent automation technologies.

While gig economies present human labor as automation and convenient services, drivers, home sharers, and micro workers exchange information about their would-be employers and develop tools to improve their economic conditions. In many instances, these makeshift support networks transform into savvy online communities that generate tangible gains. In the context of AMT, innovation arrives as scripts and browser add-ons that scan the quality of HITs. Among the most useful tools ranks the "Turkopticon," created by researcher-activists Lilly Irani and Six Silberman (Irani, Silberman 2013). In addition, reports suggest that Turkers increasingly employ bots to optimize their economic activity in AMT, resulting in quality concerns (Dreyfuss 2018). Similarly, Uber drivers have arranged mass "switch-off" operations via synchronized logouts, simulating supply shortages that trigger surge prices. Researchers explained that drivers "tried to regain some of their lost control and sense of autonomy [utilizing] forums such as UberPeople to share these stories and gain social support" (Solman 2017). Though such instances of hacking help reclaim autonomy, the challenges to the constraints of gig work also substantiate a culture of perpetual economic activity. That is, the DIY culture of support milieus reinforces the narrative of a flexible entrepreneur, an inventive and industrious gig-economy subjectivity, thus inadvertently validating gigification, taskification, and gamification.

The cultural production in these labor and resource markets is integral to their functioning. Uber and Airbnb disseminate the symbolic tools of entrepreneurialism while AMT focuses on directing representational resources toward prospective clients, particularly firms dealing in large scale data mining, intelligence, and advertising. Nevertheless, many Turkers consider themselves part of a freelance workforce, conveniently turning screen time into a profitable side hustle and escaping the grindof more conventional jobs. Workers in the gig economy are at once antagonistic, reliant, and yet rebelliously creative in the face of increasingly impenetrable forces of production. 
Critically inflected perspectives of these digital economies address blind spots in discourses on the future of work, particularly in the genre of postcapitalism.

\section{On Determinist Futures in Postcapitalist Discourses}

Critiquing postcapitalist discourses through an analysis of the gig economy is a delicate task in a time when leftist visions of the future are far and few between. Whether Margaret Thatcher's infamous dictum that "there is no alternative" or Fredric Jameson's oft-cited claim that "it has become easier to imagine the end of the world than the end of capitalism," both speak to a decline in viable alternative futures (Jameson 2003: 76). Similarly, Marc Fisher's Capitalist Realism invokes "a pervasive atmosphere, conditioning not only the production of culture but also the regulation of work and education, and acting as a kind of invisible barrier constraining thought and action" (Fisher 2012:16). Challenging a deleted future, various recent projects sketch roadmaps out of the malaise by appropriating predictions stating that automation technologies will replace large parts of the workforce. Postcapitalists envision accelerated productions, fully-automated futures, universal basic incomes, and freedom from labor.

Though postcapitalism addresses a range of problems, including socioeconomic inequality, social organization, and political formation, most authors explicitly endorse technological innovation as an indispensable component of their future schematics. Indeed, platforms, networks, sensors, and gadgets often mark the cornerstones of postcapitalist futurizing. Aside from the commendable feat of reintroducing radical visions into contemporary social and political conversations, however, postcapitalist projects warrant a critical intervention. Specifically, postcapitalism suffers from a certain naïveté, in that its authors undertheorize how emerging technologies unfold as sociotechnical systems, rather than isolated machines.

Nick Srnicek and Alex Williams' Inventing the Future: Postcapitalism and a World Without Work received an unusual amount of attention from academic and journalist outlets. Sounding a neo-Promethean clarion call to unite the left, the authors demand abandoning so-called "folk politics," whose horizontalist and localist tactics lack political efficacy (Srnicek and Williams 2016). Taking to task an unorganized contemporary left, Srnicek and Williams insist on realizing four concrete demands: "building a post-work society on the basis of fully automating the economy, reducing the working week, implementing a universal basic income, and achieving a cultural shift in the understanding of work" (Ibid.: 108). While the call for political organization around the universal principles of "synthetic freedom" barely conceals the authors' neo-Gramscian leanings, Srnicek and Williams' also present a controversial reading on the relation between technology and the future of work.

In particular, the idea of a fully automated future indicates a lack of attention to the power relations of currently existing automation processes: “Thus Inventing the Future has a sort of Wizard of Oz problem at its core. It's not clear what clever devices are behind the curtain, we're just supposed to assume that they will be sufficiently communistical if we all believe hard enough" (Galloway 2017). Srnicek and Williams conveniently conceptualize the technological advances apart from the social world, taking at face value the technologists' plugs of automation as frictionless replacements for labor.

Inventing the Future hardly accounts for the political economy of automation, that is, an articulation of how corporate providers and stakeholders, such as Google, Amazon, and the gig- economy firms make automation happen. The demand for full automation, then, ignores that the very labels of "automation," "artificial intelligence," and "on-demand networks" are part and parcel of a proprietary economy that exerts a massive influence on how such practices materialize. What is ultimately called automation is largely a function of capital valuation and corporate strategizing, which need not correspond to a technically sound meaning of the term. Amazon is not the only technology giant profiting from the shiny rhetoric of automation and AI. Google operates its AMT counterpart as Google Surveys and recently announced the rebranding of its research division into "Google AI" (Lunden 2018), furthering the mystification of crowdsourcing as quasi-machinic intelligence. In short, thinking about technologies in the domains of information (code, protocols, and "smart" algorithms), logistics (rideshare networks and "smart" cars), or material production (3-D printers), as somehow independent from capitalist flows is of limited usefulness.

Rather than heralding automation as a liberating force, it is imperative to assess how automation acts on schedules and spaces of production and, importantly, on workers' subjectivities. As Galloway clarifies, automation primarily "transforms the organic composition of labor through deskilling and proletarianization, the offshoring of menial labor, and the introduction of technical and specialist labor required to design, build, operate, and repair 
those seemingly 'automagical' machines" (Galloway 2017). This account of automation disambiguates between the fiction of automation fully replacing workers and the evidence of automation displacing workers. To recall, Uber's centrally monitored scheduling system relies on processual optimizations that increasingly prefigure when and where drivers next liquidate their time and property. Similar practices unfold in more conventional workplaces, such as Amazon fulfillment centers, where handheld tablet aids enforce specific schedules for packaging and stowing tasks. The myth of "automagical" machines belies how automating applications actually manage efficiency on the back of workers. Conjuring up the specter of automation as replacement perpetuates a long tradition of concealing the reality of displaced labor. As the image of automated services often rests on computational management of bodies in space and time, a call for more of the same appears as an unwitting embrace of capitalist structures: "it becomes difficult to untangle accelerationism from the most visionary dreams of the business elite" (Galloway 2017). In sum, there is little evidence that acceleration, be it as warehouse robotics, mobility networks, or digital labor, would mitigate the informalization, deskilling, and precarization of workers. Without a decidedly political intervention-a program Srnicek and Williams' are undeniably contributing to-accelerationism will be divided among accelerators and accelerated.

Another version is presented in Mason's Postcapitalism: A Guide to Our Future, which contends that economies generally go through 50-year-long cycles in which 25 years of economic growth precede 25 years of decline. Following Nikolai Kondratieff's theory, phases of decline are accompanied by depressions, before igniting the pursuit of new technologies, business models, markets, and money. In Mason's view, crisis theory could have predicted the depression in the 1930s and the subsequent economic upswing toward the end of the 1940s (Mason 2017). According to long-wave theory, another crisis was due in the late 1990s, if it were not for "the basic elements of the fifth long cycle: [...] network technology, mobile communications [and] a truly global marketplace and information goods" (Ibid.: 48). Somewhat confusingly, Mason also asserts that "the fourth long cycle was prolonged, distorted and ultimately broken" (Ibid.: 78), ostensibly by the same factors. Indeed, the author goes to great lengths to stress the virtues of long-wave theory, only to see it disrupted at the turn of the millennium, paving the way for a new, postcapitalist economic order based on the inherent properties of communication technologies. Replacing an economic determinism with a technological determinism, Mason spends the second half of the book exploring how the internal processes of modern information and communication platforms are "corroding market mechanisms, eroding property rights and destroying the old relationship between wages, work and profit" (Ibid.: 112).

Taking a page out of Jeremy Rifkin's The Zero Marginal Cost Society (Rifkin 2015), Mason insists, "Info goods change everything," that is, the modes in which information can be copied and distributed would undermine previous forms of value creation (Mason 2017: 116). The formula is simple: "Once you can copy and paste something, it can be reproduced for free. It has, in economic-speak, a 'zero-marginal cost”' (Ibid.: 117). Mason's presentation of information technologies remains rudimentary and, in many ways, linear. He assumes that intellectual property rights ultimately challenge the telos of value creation as conceived in conventional supply and demand models. As Christian Fuchs argues, however, "Although the copying time of information is very small, there are ways of how capital tries to institute new forms of labour-time, value creation and exploitation in the information economy. [...] information goods are not just produced once and then copied, but there are often new versions, constant updates, and forms of support labour" (Fuchs 2016: 236).

Likewise, Mason's analysis on Google's partial use of Open Source Software (OSS) illustrates his inability to apply a robust framework of political economy and to theorize technologies as continuing social environments. Mason writes, "Google is a hard-assed capitalist firm, but in the pursuit of its own interests it is forced to fight for some standards to be open and some software to be free. Google is not postcapitalist_-but as long as it keeps Android Open Source it is being forced to act in a way that prefigures non-capitalist forms of ownership and exchange” (Mason 2017: 123). Collapsing “open” and "non-capitalist," Mason ignores that technology firms engage in OSS projects to gain a competitive edge. At the heart of this misconception is Mason's view of Google as a communication firm with a popular search function. Instead, user attention contributes immensely to accumulation processes. Failing to conceptualize online advertisement, Mason's model remains reductive, as it puts a premium on the work of coders and programmers. Again, Uber drivers generate value not only by performing logistic services, but also by responding to psychological stimuli.

Mason's faith in the inherent features of technology exposes an underlying problem of theorizing openness and control as coinciding historical phenomena. While the scope of commercial outsourcing, crowdsourcing, and distributing microlabor practices already renders Mason's projection as overly confident, his determinism obfuscates the power relations in the context of OSS. A Mason-inspired analysis on Microsoft's recent acquisition of the code 
repository GitHub would, by extension, amount to yet another technology giant's turn toward postcapitalism (Finley 2018). Put differently, Microsoft's integration of GitHub would reverse the proprietary ethos in Bill Gate's infamous "Open Letter to Hobbyists," which anticipated the monopolization of operating systems and programming languages. Questioning this legacy, Mason notes that "10 percent of all corporate computers are running Linux" (Mason 2017: 122). The contrast between Gates’ proprietary software capitalism and Richard Stallman's GNU Manifesto, a milestone in the open-source movement and Linux development, however, belies the nonlinear entwinements of technology and culture. Mason's uncritical championing of OSS prematurely extrapolates essential features from a narrow technological application to a complex social context.

Since Gates' letter and Microsoft's rise to record market capitalization in 1999 (Seifert 2012), the firm has taken several steps toward incorporating OSS. For instance, Microsoft made its .Net Core (a general-purpose development platform) available to applications on Linux and OS X (Finley 2016), rendered Git (a tool to manage source code) more compatible with the Windows operating system (Finley 2012), and integrated Linux into its cloud platform Azure (Finley 2016b). These moves, however, hardly suggest a step toward a non-capitalist future. Instead, such integrative moments realize competitive strategies against other expansive networks, such as AWS and Google's AI division. The incorporation of technical openness into the flows of information capitalism resonates in responses to the Microsoft-GitHub deal. One industry analyst comments: "I think it will be good for the open-source community. I don't think Microsoft has the mentality of the early 2000s where it thought 'if you want to work with our technologies, you need to work in our ecosystem"' (Stokel-Walker 2018). Mason's insistence on a postcapitalist trajectory intrinsic to communication technologies, such as the collaborative Wikipedia library, the undeniable utility of open coding platforms, and the sharing of labor and property in the gig economy, fails to reconcile technological features of openness with core tenets of information capitalism.

The underlying problem in Mason's project could be remedied by recalling Deleuze's dictum that "machines are $[\ldots]$ social before they are technical," that is, the context in which machine function takes precedence over their narrowly defined technical processes. In Postscript, Deleuze theorizes how the rise of networks begets a kind of synchronicity of openness and control, specifically "ultrarapid forms of free-floating control" (Deleuze 1992: 4). This paradigm is synthesized in Galloway and Thacker's contention that "network control is unbothered by individuated subjects (subjected subjects). In fact, individuated subjects are the very producers and facilitators of networked control. Express yourself! Output some data! It is how distributed control functions best" (Galloway and Thacker 2007: 41). Given the coinciding of openness and control, a tentative prediction of GitHub's future might involve imagineering the social parameters of the code repository. Recalling Microsoft's purchase of the social network LinkedIn, executives might seek to increase the platform's commercialization and intensify the efficacy of popularity scores. After all, well before the acquisition, GitHub featured an effective value system rendering code collections visible to the community.

GitHub users keep up with commits (the GitHub equivalent of a code gig) flag discussions, and star projects, to indicate interest and appreciation. "Following" feature and "rockstars" label help designate popular and active users. A study exploring how the added social structure influences the repository suggests "that a new type of leadership may be emerging through follower relationship" (Blincoe et al. 2016: 38). The study clarifies that mere activity does not have the same impact, because activity is simply not publicized to the extent of popularity. Microsoft might promote an economy of social capital regarding real-time coding and the continuous publicization of these processes. In this view, GitHub would move closer toward a social network experience that meticulously registers and streams user activity. To distinguish between the technical quality of code and its represented value would become increasingly difficult. A competitive coder identity, among other traits, could be built into the programming infrastructure and reshape the GitHub experience. Indeed, transforming GitHub into a more competitive and expressive network, without formally jeopardizing its decentral appeal, might constitute a step toward turning the coding platform into an informalized labor market, another gig economy.

\section{The Continuous Production of Meaning in the Future of Work}

A nuanced criticism of postcapitalist futures, particularly how such futurizing relies on economic or technological determinism, requires a sensibility for the sociocultural and sociotechnical dynamics of late capitalist accumulation. A third contribution to postcapitalist discourse, Frase's Four Futures: Life After Capitalism, represents a hybrid project, 
in that it proposes a relatively static framework of four Weberian ideal types in which more detailed scenarios unfold. Four Futures construes thought experiments along an $\mathrm{x}$-axis, delimiting a spectrum from scarcity to abundance, and a y-axis, representing a range from egalitarianism to authoritarianism (Frase 2016). Frase, therefore, arrives at four quadrants, each representing a distinct future after capitalism.

Frase's first two scenarios take him down the path toward a future of abundance, courtesy of automation technologies, much in the same way Inventing the Future prognosticates. The latter two scenarios, meanwhile, follow current anthropogenic anxieties of climate catastrophe, leading to a future of scarcity. In each case, Frase inserts caveats of political organization, such that resources are distributed in either centralized or decentralized fashion. The resulting futures comprise "Communism" (Egalitarianism and Abundance), "Rentism” (Hierarchy and Abundance), "Socialism" (Egalitarianism and Scarcity), and "Exterminism" (Hierarchy and Scarcity). While Four Futures considers humanity's fate as contingent, an underlying utopianism permeates Frase's work, particularly concerning emergent productive and distributive capacities. Though the author guards, via the authoritarian caveat, against an inevitable end of work, he nonetheless suggests that "human societies will increasingly face the possibility of freeing people from involuntary labor" (Frase 2012). Frase, like other commentators on the left, indulges an obsession with 3-D printing and decentral organization of social production and reproduction. Four Futures, therefore, undertheorizes the extent to which differential forces of taskification, crowdsourcing strategies, gamification restructure the interfaces of work and the social meanings of productive activities. Technology remains an abstract concept, largely unaffected by the social parameters and perimeters that would guide its application in any of the four scenarios.

Frase concedes that a utopia, where socioeconomic capital no longer determines access to vital and recreational resources, might still contain hierarchies, such as social capital and popularity scores. Readers are nonetheless left wondering if the current trend toward these modes of stratification might not indeed pose the trickiest challenge to a postcapitalist project. Frase's future of a post-scarcity rentism, to be sure, addresses some of these concerns: "A characteristic of most mainstream economic discussions is their presumption that if human labor in production becomes technically unnecessary, then it will inevitably disappear. However, the system of capital accumulation and wage labor is both a technical device for efficient production and a system of power" (Frase 2016: 70). In other words, today's gig economies are already characterized by layered systems of power that exceed mere infrastructural concerns. Social market activities including the discursive production of competitive freelance subjectivities, the affective labor of gig workers in emergent spaces of taskified and gamified work, and the increasing cultural demands to liquidate resources, render contemporary economic relations, in many ways, postcapitalist.

In this context, Frase cites science fiction writer Cory Doctorow's novel Down and Out in the Magic Kingdom (Doctorow 2003), where people amass "whuffie," a currency representing sociocultural approval, inspired by the proliferation of rating systems, badges, and points on various social media. Frase misses, however, that Doctorow's point goes beyond a mere premonition of a reputation-based capitalism. Instead, Doctorow suggests that a seamless shift of all activity into quantifiable values is unfolding in the present. Doctorow's insight is therefore doubly disquieting, as the combination of corporate rent extraction with practices of establishing competitive, but largely voluntary environments already constitute the building blocks of contemporary economic forms. The roles of corporate providers in distributive labor markers are discretely normalized and largely compatible with a politics of decentralization and distributive access, as postcapitalism would have it. Doctorow offers an eerie narrative version of the Deleuzian paradigm whereby control continues to exist in accelerated movements and potential liquidations. Speculating if, when, and how a postcapitalist future might arrive seems less productive than theorizing how sociotechnical interfaces and mutable subjectivities already convey the appearance of such a future in the present.

Doctorow imagines several futures that defamiliarize the teloi of supposedly clear-cut technologies and fixed political systems by introducing ambivalent social dynamics, idiosyncratic cultural conventions, and rather heterogeneous protagonists. Doctorow's latest novel, Walkaway, delimits the matter more succinctly (Doctorow 2017). Compared to Srnicek and Williams' "fully-automated future," Mason's "zero-marginal-cost-techno-utopia," and Frase's somewhat static ideal typography of future imaginaries, Doctorow refuses to adopt to the classic categories of utopian and dystopian fiction:

[Walkaway is] the story of a utopia in progress, as messy as every new thing ever is, told in the form of people talking to each other, arguing with each other and working together to solve problems. It's all about the deep, disturbing, recognizable weirdness of the future that must come from the present we have already made for ourselves, trying to figure out what went wrong and what comes next (Sheelan 2017).

Walkaway takes seriously the notion that technologies are continuously subject to application and interpretation. 
Given its emphasis on social interaction and contingency, it is not clear whether Walkaway takes place in a utopian or dystopian setting, a tension that produces a simple but effective narrative effect of contracting a comfortable distance that science fiction tends to offer, replacing it with a lower suspension of disbelief. As Doctorow notes, "The most perfect society will exist in an imperfect universe, one where the second law of thermodynamics means that everything needs constant winding up and fixing and adjusting" (Doctorow 2017b). The emphasis on an imperfect world, where constant updates are required, challenges the sterile futures of postcapitalism and their presentation of technologies as asocial, perhaps even ending the social. As the discussion intimates, such visions derive from rudimentary understandings of economies as merely accruing capital value and technologies as simply performing their desired functions. Instead, today's modes of accumulation deal in power, identity, and subjectivity.

One Walkaway character conveys this insight strikingly: Limpopo, a manager at the "Belt and Brace," a bed and breakfast type place in the badlands of an uninviting civilization controlled by a wealthy elite and the looming dread of "non-work," a post-scarcity phenomenon that speaks to an ambiguous prospect of automation. Limpopo helped create the bed and breakfast from scavenged waste and advanced coding and printing software, evoking an image of a versatile structure that blends material and immaterial features of Airbnb and GitHub. The distance to civilization does not undo the blurry lines between work and play so familiar from the gig economy, though the "Belt and Brace" is clearly modeled around a futuristic version of a communist enclave. Limpopo is introduced as a feeling of two minds about her stellar commits record, which charts user repairs and improvements to the building infrastructure:

In a gift economy, you gave without keeping score, because keeping score implied an expectation of reward. If you're doing something for a reward, it's an investment, not a gift. [...] It was so easy to keep score, the leaderboard was so satisfying that she couldn't help herself. She wasn't proud of this. Mostly (Doctorow 2017: 44).

The short passage illustrates the organic social interactions at the intersection of work and play that would likely remain intact in a postcapitalist future. Moreover, the passage illustrates the ease whereby gig economy providers incorporate and manipulate social relations, affecting the production of a subjectivity constantly involved in economic activity. Programming interfaces to integrate seamlessly with everyday activity constitutes an efficient strategy to prolong user engagement with gigs, commits, HITs, shares, and so on.

\section{| Conclusion}

Despite the criticism in this chapter, developing the discourse on postcapitalism is crucial, as few other genres currently attempt to elevate work-related issues in a politically organized fashion. As an important caveat to this claim, contributors to postcapitalism are advised to question their preoccupation with a technology-induced future of fully automated production or evenly distributed services. Instead, postcapitalism must be informed by presentday accounts on labor dynamics ranging from fulfillment centers to online crowdsourcing. While the general sense that work is undergoing drastic changes is uncontroversial, automation processes and networked distribution of services are part and parcel of highly differential enterprises that involve workers in continuous market activity. The effect that seems to elide the registers of postcapitalist thinkers is the simultaneous production of vertical power relations that favor providers but increasingly disenfranchise gig workers.

This mode of social control requires theorizing beyond mere modes of exploitation toward a more open and subjective paradigm, where asymmetrical and precarious working conditions register increasingly as freelance opportunities. In this paradigm, economic imperatives and autonomy are mediated by interfaces that blend categories of work and play. Thus, further study is required to explore the shadowy spaces of partial automation in highly informalized work environments of which the colloquial gig economy is but one manifestation of a larger trajectory of integrating quasi-autonomous subjectivities into the machinic circuits of platform providers. The gig economy has evolved into a blend of corporeal and incorporeal resource markets that comprise the management of human bodies and their immediate assemblages. The cultural economy to participate in such systems is sustained by an injunction of continuous engagement and a pervasive demand to liquidate idle resources. The future of work is eclipsed by an endless present of constant enterprising across numerous platforms. 


\section{References}

Atkinson, Robert D and John Wu. 2017. "False Alarmism: Technological Disruption and the U.S. Labor Market, 18502015." Information Technology \& Innovation Foundation. 1-28.

Arntz, Melanie, Terry Gregory, and Ulrich Zierahn. 2016. "The Risk of Automation for Jobs in OECD: A Comparative Analysis." OECD Social, Employment and Migration Working Papers, no. 189.

Berry, David M. 2015. Critical Theory and the Digital. New York: Bloomsbury.

Blincoe, Kelly Sheoran Jyoti, Sean Goggins, Eva Petakovic, and Daniela Damian. 2016. "Understanding the popular users: Following, affiliation influence and leadership on GitHub" Information and Software Technology. Vol. 70 (February): 30-39. DOI: https://doi.org/10.1016/j.infsof.2015.10.002

Boltanski, Luc, and Eve Chiapello. 2007. The New Spirit of Capitalism. London: Verso, 2007.

Brynjolfsson, Erik and Andrew McAfee. 2014. The Second Machine Age: Work, Progress, and Prosperity in a Time of Brilliant Technologies. New York: W.W. Norton \& Company, Inc.

Chen, Brian X. 2017. "The Guide to Being an Airbnb Superhost." The New York Times. Accessed 7.22.2018 at https://www. nytimes.com/2017/01/11/technology/personaltech/the-guideto-being-an-airbnb-superhost.html.

Crary, Jonathan. 2014. 24: Late Capitalism and the Ends of Sleep. London: Verso.

Deleuze, Gilles. 1992. "Postscript on the Societies of Control." October. Vol 59 (Winter): 3-7.

Dickey, Megan Rose. 2017. "Uber is partially banned in Tel Aviv.” TechCrunch. Accessed 8.2.2018 at https://techcrunch. com/2017/11/27/uber-is-partially-banned-in-tel-aviv/.

Doctorow, Cory. 2017. "Disasters Don't Have to End in Dystopias" Wired. Accessed 8.11.2018 at https://www.wired.com/2017/04/ cory-doctorow-walkaway/. Tor.

2003. Down and Out in the Magic Kingdom. New York:

2017. Walkaway. New York: Tor.

Finley, Klint. 2018a. "Microsoft's GitHub Deal is its Latest Shift From Windows." Wired. Accessed 8.29.2018 at https://www. wired.com/story/microsofts-github-deal-is-its-latest-shift-from -windows/.

. 2016a. "Microsoft Says It's in Love with Linux. Now It's Finally Proving It." Wired. Accessed 8.29.2018 at https://www. wired.com/2016/06/microsofts-open-source-love-affair-reaches -new-heights/.
2012. "Microsoft Cradles Linus Torvalds' (Other) Baby." Wired. Accessed 8.29.2018 at https://www.wired.com/2013/01 /microsoft-announces-support-for-linus-other-baby/.

2016b. "Now You Can Use Linux Just Like Microsoft Does." Wired. Accessed 8.29.2018 at https://www.wired. com/2016/03/now-can-use-linux-just-like-microsoft/.

Frase, Peter. 2016. Four Futures: Visions of the World After Capitalism. London: Verso.

. 2011. "Four Futures." Jacobin. Accessed 7.10.2018 at https://www.jacobinmag.com/2011/12/four-futures.

Ford, Martin. 2016. Rise of the Robots: Technology and the Threat of a Jobless Future. New York: Basic Books.

Fowler, Susan. 2018. "'What Have We Done?': Silicon Valley Engineers Fear They've Created A Monster." Vanity Fair, Accessed 8.25.2018 at https://www.vanityfair.com/news/2018 /08/silicon-valley-engineers-fear-they-created-a-monster.

Fuchs, Christian. 2016. "Henryk Grossmann 2.0: A Critique of Paul Mason's Book 'PostCapitalism: A Guide to the Future." tripleC: Communication, Capitalism \& Critique. Vol 14.1: 232-243. DOI https://doi.org/10.31269/triplec.v14i1.757.

Galloway, Alexander. 2012. The Interface Effect. Cambridge: Polity.

. 2017 "Brometheanism," Cultureandcommunication. Accessed 8.22.2018 at http://cultureandcommunication.org/ galloway/brometheanism.

. and Eugene Thacker. 2007. The Exploit: A Theory of Networks. Minneapolis: University of Minnesota Press.

Irani, Lilly. 2016. “The Hidden Faces of Automation," XRDS Vol. 23.2 (Winter): 34-37. DOI 10.1145/3014390.

Jameson, Fredric. 2003. "Future City." New Left Review Vol. 21 (May-June): 65-79.

Katz, Miranda. 2017. "Amazon's Turker Crowd Has Had Enough." Wired. Accessed 8.5.2018 at https://www.wired.com/story/ amazons-turker-crowd-has-had-enough/.

Katz, Lawrence F. and Alan B. Krueger. 2016. “The Rise and Nature of Alternative Work Arrangements in the United States, 1995-2015." NBER Working Paper Series. 2-47.

Lunden, Ingrid. 2018. "Google goes all-in on artificial intelligence, renames research division Google AI." TechCrunch. Accessed 8.14.208 at https://techcrunch.com/2018/05/08/google-goes-all-in-on-artificial-intelligence-renames-research-division-google-ai/.

Mason, Paul. Postcapitalism: A Guide to Our Future. 2017. New York: Farrar, Straus and Giroux. 
Mason, Sarah. 2018. "High Score, Low Pay: Why the Gig Economy Loves Gamification.” The Guardian. Accessed 11.28.2018 at https://www.theguardian.com/business/2018/nov/20/highscore-low-pay-gamification-lyft-uber-drivers-ride-hailing-gigeconomy.

Perez, Sarah. 2016. "Airbnb revamps its app with new tools for hosts, improved messaging," TechCrunch. Accessed 7.22.2018 at https://techcrunch.com/2016/09/27/airbnb-revamps-its-appwith-new-tools-for-hosts-improved-messaging/.

Rhodes, Margaret. 2015. “Airbnb's Redesigned Tools Make it Easier to Be a Good Host." Wired. Accessed 7.22.2018 at https://www .wired.com/2015/11/airbnbs-redesigned-tools-make-it-easier-to -be-a-good-host/.

Rifkin, Jeremy. 2015. The Zero Marginal Cost Society. Basingstoke: Palgrave Macmillan.

Schwab, Klaus. 2016. The Fourth Industrial Revolution. Geneva: World Economic Forum.

Sennett, Richard. 2007. The Culture of the New Capitalism. London: Yale University Press.

Seifert, Dan. 2012. “Apple beats Microsoff's 1999 market cap record." The Verge. Accessed 8.5.2018 at https://www.theverge. com/2012/8/20/3255593/apple-now-most-valuable-company-ever-overtaking-1990s-microsoft.
Sheelan, Jason. 2017. "In 'Walkway', A Blueprint For A New, Weird (But Better) World.” NPR. Accessed 8.11.2018 at https://www. npr.org/2017/04/27/523587179/in-walkaway-a-blueprint-for -a-new-weird-but-better-world.

Solman, Paul. 2017. "How Uber drivers game the app and force surge pricing." PBS. Accessed 6.5.2018 at https://www.pbs.org/ newshour/economy/uber-drivers-game-app-force-surge-pricing.

Solon, Olivia. 2018. "The rise of 'pseudo-AI': how tech firms quietly use humans to do bots' work." The Guardian, Accessed 8.30.2018 at https://www.theguardian.com/technology/2018/ jul/06/artificial-intelligence-ai-humans-bots-tech-companies.

Srnicek, Nick and Alex Williams. 2016. Inventing the Future: Postcapitalism and a World Without Work. London: Verso.

Stokel-Walker, Chris. 2018. "Microsoft is buying GitHub, but will the open source world follow?" Wired. Accessed 8.29.2018 at http://www.wired.co.uk/article/ microsoft-github-deal-open-source.

Sundararajan, Arun. 2016. The Sharing Economy: The End of Employment and the Rise of Crowd-Based Capitalism. Cambridge: The MIT Press.

Vinik, Danny. 2018. “The Real Future of Work.” Politico. Accessed 8.22.2018 at https://www.politico.com/magazine/ story/2018/01/04/future-work-independent-contractors-alternative-work-arrangements-216212. 
